\title{
METODOLOGÍA PARA LA SELECCIÓN Y PRIORIZACIÓN DE YACIMIENTOS DE CRUDO LIVIANO CANDIDATOS A LA INYECCIÓN DE AIRE: CASO DE APLICACIÓN
}

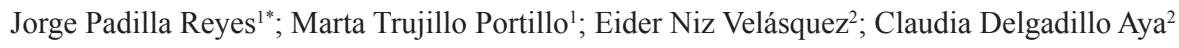 \\ ${ }^{1}$ Grupo de Investigación Recobro Mejorado. Universidad Industrial de Santander (UIS). Carrera 27 Calle 9. Bucaramanga, \\ Colombia. \\ ${ }^{2}$ Instituto Colombiano del Petróleo. Vía Piedecuesta Km 7. Piedecuesta, Colombia. \\ *E-mail: jorge.padilla1@hotmail.com
}

\section{RESUMEN}

Los procesos de recobro térmico han sido estudiados, implementados y mejorados durante varios años. A finales de los 90's se despertó un gran interés en la inyección de aire en yacimientos de crudo liviano, producto de la implementación exitosa de un procesos denominado High Pressure Air Injection (HPAI), en el Campo Buffalo (USA), que es una variante del proceso de inyección de aire en crudos livianos, especial para yacimientos profundos con bajas permeabilidades y porosidades. Prueba de esto son el Campo West Hackberry (USA), más de cinco proyectos comerciales a lo largo de la Cuenca Williston (USA) y un piloto en el Campo Zhongyuan (China). Adicionalmente se han realizado estudios de factibilidad en México y Argentina. Este artículo presenta un caso de aplicación de la metodología de priorización para la identificación de yacimientos de crudo liviano candidatos a la inyección de aire.

Palabras clave: Inyección de aire, Yacimientos de Crudo Liviano, Recuperación Mejorada de Petróleo.

\section{METHODOLOGY FOR SCREENING AND PRIORITIZATION OF LIGHT OIL RESERVOIRS CANDIDATES TO AIR INJECTION: IMPLEMENTATION CASE}

\begin{abstract}
Thermal enhanced oil recovery process has been studied, implemented and improved over the years. In the late 90's there has been significant interest in the light oil air injection (LOAI) process, since the successful implementation of the process known as High Pressure Air Injection in the Buffalo Field (USA), which is a variation from the air injection in light oil reservoirs, applicable to deep reservoirs with low permeability and porosity. Proof of this are the West Hackberry Field (USA), more than five commercial projects along the Williston Basin (USA) and a pilot in the Zhongyuan Field (China). Additionally, feasibility studies have been also been performed in Mexico and Argentina. This paper shows an implementation case of a methodology proposed previously for prioritization of light oil reservoir candidates to air injection.
\end{abstract}

Keywords: Air Injection, Light Oil Reservoirs, Enhanced Oil Recovery.

Cita: Padilla, J., Trujillo, M., Niz, E. y Delgadillo, C. (2017). Metodología para la selección y priorización de yacimientos de crudo liviano candidatos a la inyección de aire: caso de aplicación. Revista Fuentes: El reventón energético, 15 (1), 41-57. 


\section{INTRODUCCIÓN}

La inyección de aire en crudo liviano (LOAI - Light Oil Air Injection) es una tecnología emergente para recobro de este tipo de fluidos. En el proceso se inyecta aire comprimido esperando que el oxígeno presente en el aire inyectado reaccione con parte del aceite del yacimiento, aumentando la temperatura y produciendo gases de combustión, los cuales realizan un barrido del crudo desde la zona de reacción hasta los pozos productores. La mezcla gas-aceite puede ser inmiscible, parcialmente miscible o miscible (Montes, Gutierrez, Moore, Mehta y Ursenbach, 2010).

Antes de implementar cualquier técnica EOR, es necesario realizar un estudio de screening que sugiera que el yacimiento candidato es apto para la aplicación de determinado proceso. Los estudios de screening evitan los altos costos ligados a estudios de factibilidad económica y descripción detallada del yacimiento. Un set de criterios de screening generalmente incluye: 1) Características del yacimiento, 2) Reservas remanentes, 3) Estabilidad del proceso de inyección y 4) Simulación numérica conceptual.

Debido al naciente interés en la inyección de aire en yacimientos de crudo liviano; producto del éxito alcanzado en diferentes proyectos a lo largo de la cuenca Williston (USA), Campo West Hackberry (USA) y recientemente un piloto en la China; Niz-Velásquez, Tujillo, Delgadillo y Padilla (2014) plantearon una metodología secuencial para la priorización de yacimientos candidatos a LOAI, basada en criterios de screening, analogías y la evaluación de algunos efectos físicos de primer orden evaluados a partir de simulación numérica. En este artículo se presenta la aplicación de la metodología de priorización planteada en 2014 sobre un grupo de yacimientos hipotéticos.

\section{INYECCIÓN DE AIRE EN YACIMIENTOS DE CRUDO LIVIANO}

El objetivo principal de las reacciones de oxidación en un proceso LOAI es producir gases de combustión para mejorar la recuperación de aceite mediante el barrido por estos gases (Greaves, Ren y Rathbone, 1998). En este tipo de procesos la tasa de inyección de aire es mucho menos importante que para aun proceso de Combustión In Situ (inyección de aire en aceites pesados), dada la mayor reactividad del hidrocarburo liviano. En la Figura 1 se muestra una representación conceptual de un proceso de inyección de aire. Los gases de combustión producidos durante el proceso, son principalmente una mezcla de $\mathrm{CO}_{2} \mathrm{y} \mathrm{N}_{2}$, los cuales realizan un barrido del aceite hacia los pozos productores bajo la combinación de varios mecanismos.

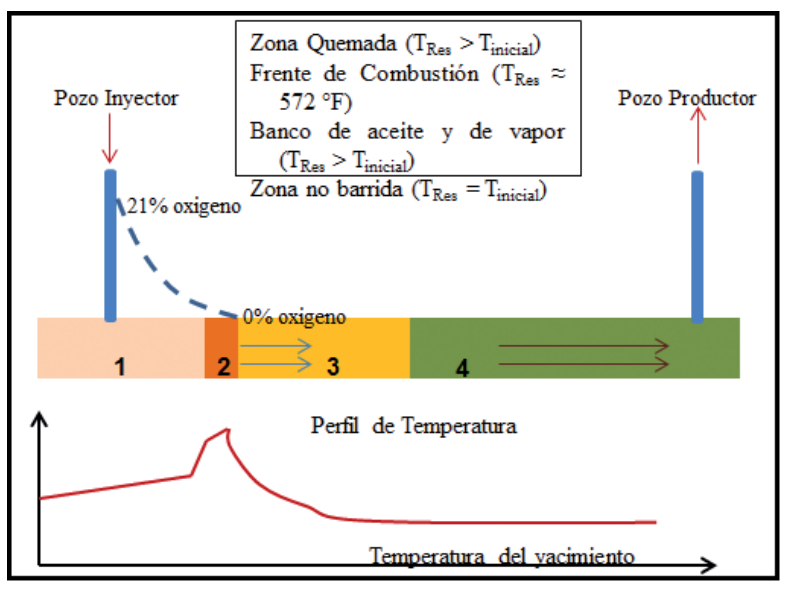

Figura 1. Representación de un proceso de inyección de aire en crudos livianos. (Hughes y Sarma, 2006).

Dado que para un proceso LOAI, las reacciones de oxidación son mucho menos sensibles al flujo de aire, el proceso se puede mantener en el modo de ruptura de enlace (las reacciones de oxidación solo producen óxidos de carbono y agua), mientras que están operando a tasas moderadas de inyección de aire en yacimientos que presentan grandes espaciamientos entre pozos. Incluso si el flujo de aire no es suficiente para mantener el proceso en el modo de ruptura de enlace, continuará el flujo de gas en aquellas zonas que no han sido afectadas por las reacciones de oxidación.

En teoría, los procesos LOAI pueden operar hasta que la relación aire inyectado/ aceite producido (AOR) alcance el limite económico o cuando el contacto entre la corriente de aire y el aceite, no sea lo suficientemente prolongado para que la totalidad del oxígeno sea removido antes de alcanzar los pozos productores.

Cuando se inyecta aire en un yacimiento de petróleo, ocurren dos fenómenos simultáneamente: desplazamiento y oxidación del aceite. De acuerdo con la eficiencia del desplazamiento y la intensidad de la oxidación, cuatro tipos de procesos pueden ocurrir (Turta y Singhal, 2001).

- Desplazamiento inmiscible de aire (IAF - Inmiscible Air Flooding) con altas temperaturas de oxidación.

- IAF con bajas temperaturas de oxidación.

- Desplazamiento miscible de aire (Miscible Air Flooding) con régimen de oxidación de altas temperaturas.

- MAF con bajas temperaturas de oxidación. 


\section{METODOLOGÍA DE PRIORIZACIÓN}

Partiendo del hecho de que para el caso particular de la inyección de aire en crudo liviano el número de proyectos con información disponible del desempeño no alcanza para efectuar una correlación estadísticamente significativa con los parámetros de yacimiento, y menos aun considerando la incertidumbre asociada con cada valor reportado, se recomienda emplear la metodología propuesta por Niz-Velázquez et al. (2014) para la priorización y selección de los yacimientos de crudo liviano con mayor potencial para soportar un proceso de inyección de aire. En general la metodología plantea:

- Screening ponderado: Consiste en la clasificación cualitativa del grado de proximidad de un yacimiento respecto a los criterios de screening: Transmisibilidad $(\mathrm{kh} / \mu)$, Concentración de aceite $\left(S_{o} \varphi\right)$, Temperatura inicial y profundidad (Tabla 1). El yacimiento que cumpla a cabalidad con los criterios tendrá un total de 10 puntos.

- Grado de Similitud - Analogías: Esta etapa se realiza con el objetivo de determinar el grado de similitud de un yacimiento candidato respecto a yacimientos con aplicación exitosa de LOAI (Tabla 2), analizando estadísticamente ocho propiedades y clasificándolas según corresponda en tres categorías así: alta similitud (1.0), similitud intermedia (0.5), baja similitud (0.0). Un yacimiento podrá obtener un máximo de 8 puntos.

Posterior a la implementación del screening ponderado y la evaluación del grado de similitud sobre cada uno de los yacimientos estudiados, se consolida un ranking preliminar, que dará una primera aproximación sobre los yacimientos con mayor potencial para la aplicación de un proceso LOAI. En esta etapa es muy probable que varios yacimientos obtengan un mismo puntaje en el screening ponderado, por lo tanto el criterio de desempate será el grado de similitud más alto frente a uno de los yacimientos tipo.

- Priorización por simulación numérica: Luego de construir el ranking preliminar, se procede a priorizar mediante el uso de la simulación numérica. La simulación numérica requiere de una mayor cantidad de datos que el screening binario. Además, el simular un proceso de inyección de aire en crudos livianos puede llegar a ser muy complejo, si se incluyen efectos térmicos, cinéticos, composicionales y/o de cambio de presión. Por lo tanto, para la priorización de los yacimientos con potencial para la implementación de LOAI basta con la evaluación de efectos físicos de primer orden, inyectividad (modelos 1D) y la estabilidad del desplazamiento inmiscible teniendo en cuenta los efectos de la segregación gravitacional (modelos 2D).

Tabla 1. Ponderación para la priorización por screening.

\begin{tabular}{|c|c|c|c|c|c|c|c|c|}
\hline \multirow{2}{*}{\multicolumn{2}{|c|}{ Parámetro }} & \multirow{2}{*}{ Peso } & \multicolumn{2}{|c|}{ Categoría 1} & \multicolumn{2}{|c|}{ Categoría 2} & \multicolumn{2}{|c|}{ Categoría 3} \\
\hline & & & Rango & Puntaje & Rango & Puntaje & Rango & Puntaje \\
\hline Transmisibilidac & $\mathrm{kh} / \mu$ & 3.0 & $20-300$ & 0.5 & $300-3000$ & 1.0 & $>3000$ & 0.5 \\
\hline Concentración d & aceite & 2.5 & $0.08-0.12$ & 0.33 & $0.12-0.17$ & 0.67 & $>0.17$ & 1.0 \\
\hline \multirow{2}{*}{ Espesor neto, $\mathrm{ft}$} & $<66 \mathrm{ft}$ & \multirow{2}{*}{2.0} & $<10$ & 0.5 & $10-40$ & 1.0 & $>40$ & 0.5 \\
\hline & $>66 \mathrm{ft}$ y Buzamiento $>10^{\circ}$ & & - & - & $<100$ & 1.0 & $>100$ & 0.5 \\
\hline Temperatura ini & ial, ${ }^{\circ} \mathrm{F}$ & 1.5 & $125-165$ & 0.33 & $<100$ & 0.67 & $>205$ & 1.0 \\
\hline Profundidad, $\mathrm{ft}$ & & 1.0 & $3000-7000$ & 0.5 & $165-205$ & 1.0 & $>11000$ & 0.5 \\
\hline Total & & 10.0 & & & $7000-11000$ & & & \\
\hline
\end{tabular}

Tabla 2. Grupos de yacimientos tipo, con aplicación exitosa del proceso LOAI

\begin{tabular}{lcccc}
\hline Parámetro & $\begin{array}{c}\text { TIPO 1 } \\
\text { Williston Basin }\end{array}$ & $\begin{array}{c}\text { TIPO 2 } \\
\text { Brea-Olinda, May-Libby, Fosterton }\end{array}$ & $\begin{array}{c}\text { TIPO 3 } \\
\text { Heidelberg, Sloss }\end{array}$ & $\begin{array}{c}\text { TIPO 4 } \\
\text { West Hackberry }\end{array}$ \\
\hline Profundidad, ft & $8,000-10,000$ & $3,400-6,200$ & $6,200-11,500$ & $7,500-9,000$ \\
Presión, psi & $3,500-4,200$ & $50-800$ & $1,500-2,300$ & $2,500-3,300$ \\
Transmisibilidad, $\mathrm{mD}-\mathrm{ft} / \mathrm{cP}$ & $50-250$ & $2,000-3,400$ & $260-3,400$ & 30,000 \\
Gravedad API $^{\text {Buzamiento, }}{ }^{\circ}$ & $30-39$ & $22-40$ & $24-39$ & 30 \\
Temperatura, $^{\circ} \mathrm{F}$ & $0-3$ & $0->25$ & $0-15$ & $>60$ \\
Concentración de Aceite $^{\text {Espesor neto, } \mathrm{ft}}$ & $215-230$ & $125-135$ & $200-220$ & $174-200$ \\
\hline
\end{tabular}


Tabla 3. Yacimientos Candidatos a la implementación de un proceso LOAI.

\begin{tabular}{|c|c|c|c|c|}
\hline Parámetro & $\begin{array}{c}\text { Yacimiento } \\
\mathbf{A}\end{array}$ & $\begin{array}{c}\text { Yacimiento } \\
\text { B }\end{array}$ & $\begin{array}{c}\text { Yacimiento } \\
\text { C }\end{array}$ & $\begin{array}{c}\text { Yacimiento } \\
\text { D }\end{array}$ \\
\hline Profundidad, $\mathrm{ft}$ & 6900 & 9700 & 6800 & 8300 \\
\hline Presión, psi & 1580 & 2400 & 1470 & 3500 \\
\hline $\begin{array}{l}\text { Transmisibilidad, } \\
\mathrm{mD}-\mathrm{ft} / \mathrm{cP}\end{array}$ & 3150 & 16000 & 335 & 12000 \\
\hline Gravedad API & 30 & 31 & 21 & 27 \\
\hline Buzamiento, ${ }^{\circ}$ & - & 12.5 & 37.5 & 5 \\
\hline Temperatura, ${ }^{\circ} \mathrm{F}$ & 207 & 230 & 143 & 204 \\
\hline $\begin{array}{l}\text { Concentración de } \\
\text { Aceite }\end{array}$ & 0.18 & 0.09 & 0.10 & 0.08 \\
\hline Espesor neto, $\mathrm{ft}$ & 15 & 36 & 105 & 35 \\
\hline
\end{tabular}

\section{APLICACIÓN DE LA METODOLOGÍA DE PRIORIZACIÓN}

En esta sección se presentan los resultados obtenidos tras la implementación de la metodología de priorización propuesta por Niz-Velásquez et al. (2014), sobre un grupo de cuatro yacimientos hipotéticos cuyas propiedades son presentadas en la Tabla 3.

\section{PRIORIZACIÓN POR SCREENING PONDERADO Y GRADO DE SIMILITUD}

Según lo presentado en la sección anterior y lo descrito por Niz-Velásquez et al. (2014), se aplicó el screening ponderado sobre la información de los yacimientos presentados en la Tabla 3, encontrando que el Yacimiento $A$ con base en los criterios de screening es el que presenta las mejores características para la implementación de un proceso de LOAI (Tabla 4).

Considerando que no hubo puntajes iguales en la etapa de priorización por screening ponderado, no sería necesario evaluar el nivel de similitud de los yacimientos bajo estudio frente a los yacimientos tipo. Sin embargo, en la Tabla 5 se presenta el grado de similitud de cada uno de los yacimientos respecto a los yacimientos tipo. En ella se aprecia claramente, que los cuatro yacimientos estudiados se asemejan en mayor medida al yacimiento Tipo 3 que corresponde a un yacimiento con valores moderados de transmisibilidad y presión. Finalmente, la Tabla 6 presenta el consolidado con priorización por screening ponderado y grado de similitud.
Tabla 4. Resultados aplicación screening ponderado.

\begin{tabular}{|c|c|c|c|c|}
\hline Parámetro & $\begin{array}{c}\text { Yacimiento } \\
\text { A }\end{array}$ & $\begin{array}{c}\text { Yacimiento } \\
\text { B }\end{array}$ & $\begin{array}{c}\text { Yacimiento } \\
\mathrm{C}\end{array}$ & $\begin{array}{c}\text { Yacimiento } \\
\text { D }\end{array}$ \\
\hline $\begin{array}{c}\text { Transmisibilidad, } \\
\mathrm{kh} / \mu\end{array}$ & 1.5 & 1.5 & 3.0 & 1.5 \\
\hline $\begin{array}{l}\text { Concentración de } \\
\text { aceite }\end{array}$ & 2.5 & 0.83 & 0.83 & 0.83 \\
\hline \multirow{2}{*}{$\begin{aligned} \text { Espesor } & <66 \mathrm{ft} \\
\text { neto, } \mathrm{ft} & >66 \mathrm{ft}\end{aligned}$} & 2.0 & 2.0 & - & 2.0 \\
\hline & - & - & 1.0 & - \\
\hline $\begin{array}{l}\text { Temperatura } \\
\text { inicial, }{ }^{\circ} \mathrm{F}\end{array}$ & 1.5 & 1.5 & 0.5 & 1.0 \\
\hline Profundidad, $\mathrm{ft}$ & 0.5 & 1.0 & 0.5 & 1.0 \\
\hline Total & 8.0 & 6.83 & 5.83 & 6.33 \\
\hline
\end{tabular}

Tabla 5. Grado de similitud de los yacimientos evaluados respecto a los yacimientos tipo.

\begin{tabular}{lcccc}
\hline & $\begin{array}{c}\text { TIPO 1 } \\
\text { Williston } \\
\text { Basin }\end{array}$ & $\begin{array}{c}\text { TIPO 2 } \\
\text { Brea-Olinda, } \\
\text { May-Libby, } \\
\text { Fosterton }\end{array}$ & $\begin{array}{c}\text { TIPO 3 } \\
\text { Heidelberg, } \\
\text { Sloss }\end{array}$ & $\begin{array}{c}\text { TIPO 4 } \\
\text { West } \\
\text { Hackberry }\end{array}$ \\
\hline Yacimiento A & 2.5 & 4.0 & 5.5 & 3.0 \\
\hline Yacimiento B & 3.5 & 2.0 & 3.5 & 3.5 \\
\hline Yacimiento C & 2.0 & 3.0 & 4.0 & 2.0 \\
\hline Yacimiento D & 2.5 & 2.0 & 4.5 & 4.0 \\
\hline
\end{tabular}

Tabla 6. Consolidado priorización por screening ponderado y grado de similitud.

\begin{tabular}{ccccc}
\hline Prioridad & Yacimiento & $\begin{array}{c}\text { Puntaje } \\
\text { Screening }\end{array}$ & $\begin{array}{c}\text { Yacimiento } \\
\text { Análogo }\end{array}$ & $\begin{array}{c}\text { Grado de } \\
\text { similitud }\end{array}$ \\
\hline $\mathbf{1}$ & Yacimiento A & 8.0 & Tipo 3 & 5.5 \\
$\mathbf{2}$ & Yacimiento B & 6.8 & Tipo 3 & 3.5 \\
$\mathbf{3}$ & Yacimiento D & 6.3 & Tipo 3 & 4.5 \\
$\mathbf{4}$ & Yacimiento C & 5.8 & Tipo 3 & 4.0 \\
\hline
\end{tabular}

\section{PRIORIZACIÓN POR SIMULACIÓN NUMÉRICA}

En la priorización por simulación numérica conceptual se busca evaluar la inyectividad, estabilidad y el factor de recobro de un proceso de inyección de gas inmiscible. Para ello se usan modelos de simulación 1D y 2D. Para la construcción de los diferentes modelos de simulación se requiere: información PVT, curvas de permeabilidad relativa y propiedades promedio del yacimiento. Estas propiedades son presentadas en la sección de anexos.

\section{RANKING DE INYECTIVIDAD}

Para evaluar la inyectividad de los campos candidatos a LOAI se construyeron modelos 1D (Figura 2), con un espaciamiento entre el pozo inyector y productor de 1000 metros, 500 metros de ancho y el espesor reportado en la Tabla 3. Se estableció un tiempo de simulación de 
10 años y se consideraron dos escenarios: En el primero se inyectó aire a una presión de 400 psia por encima de la presión actual del yacimiento y en el segundo 1000 psia por encima. Lo anterior se realizó con el objetivo de evaluar el efecto de la re-presurización.

Como parámetros de control en el pozo productor se consideraron BHP mínima igual a la presión actual del yacimiento y un máximo Draw Down de 200 psia. En el pozo inyector los parámetros de control fueron una BHP máxima (400 psia o 1000 psia por encima de la presión actual del yacimiento según el caso) y la tasa máxima de inyección de gas 1 MMSCF (en todos los modelos el gas inyectado fue Nitrógeno).

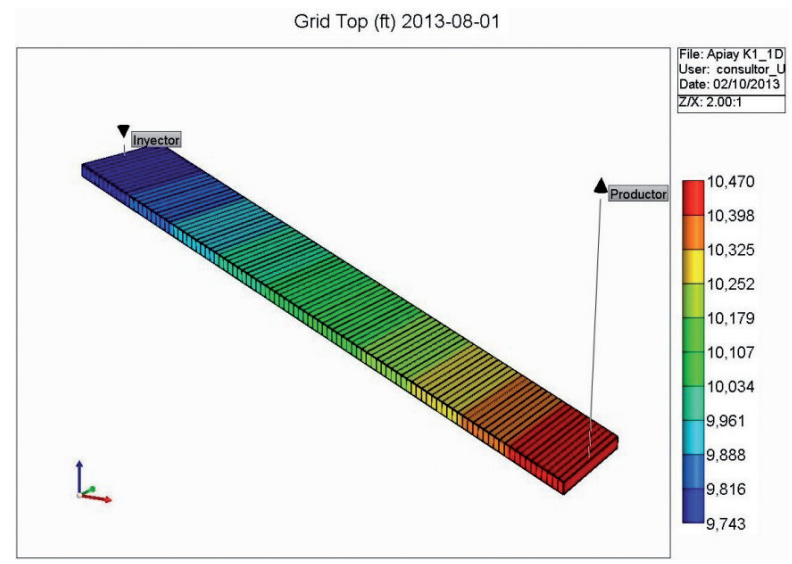

Figura 2. Esquema modelo 1D para evaluar la inyectividad.

Para definir el número de celdas óptimo se realizaron corridas con modelos de diferentes tamaños $(300,500$, 800, 1000 celdas), determinando que el número óptimo de celdas sería aquel en el que los resultados arrojados por el simulador no variaran significativamente con respecto a un modelo de menor tamaño. En la Tabla 7 se presenta el numero óptimo de celdas para cada modelo 1D.

Tabla 7. Número óptimo de celdas modelos 1D.

\begin{tabular}{cc}
\hline Yacimiento & Número óptimo de celdas \\
\hline Yacimiento A & 500 \\
\hline Yacimiento B & 1000 \\
\hline Yacimiento C & 800 \\
\hline Yacimiento D & 1000 \\
\hline
\end{tabular}

En las Tablas 8 y 9, se presentan los rankings de inyectividad con y sin efecto de re-presurización. Se reporta el volumen inyectado a condiciones de yacimiento por pie de formación, para evitar darle ventaja a los yacimientos con espesores grandes. En estas dos tablas se ve claramente que el yacimiento con las mejores condiciones de inyectividad es el Yacimiento
$A$, considerando su bajo espesor, la relativamente baja presión del yacimiento y su alta transmisibilidad.

Tabla 8. Ranking inyectividad con efectos de represurización.

\begin{tabular}{cccc}
\hline Prioridad & Yacimiento & $\begin{array}{c}\text { Gas inyectado } \\
\text { acumulado } \\
\text { 10 años, MMCF }\end{array}$ & $\begin{array}{c}\text { Gas inyectado, } \\
\text { MMCF/ft }\end{array}$ \\
\hline $\mathbf{1}$ & Yacimiento A & 29.07 & 1.94 \\
$\mathbf{2}$ & Yacimiento D & 30.55 & 0.87 \\
$\mathbf{3}$ & Yacimiento B & 26.51 & 0.74 \\
$\mathbf{4}$ & Yacimiento C & 2.01 & 0.02 \\
\hline
\end{tabular}

Tabla 9. Ranking inyectividad sin efectos de represurización.

\begin{tabular}{cccc}
\hline Prioridad & Yacimiento & $\begin{array}{c}\text { Gas inyectado } \\
\text { acumulado } \\
\text { @ } \mathbf{1 0} \text { años, MMCF }\end{array}$ & $\begin{array}{c}\text { Gas inyectado, } \\
\text { MMCF/ft }\end{array}$ \\
\hline $\mathbf{1}$ & Yacimiento A & 29.07 & 1.94 \\
$\mathbf{2}$ & Yacimiento D & 31.33 & 0.90 \\
$\mathbf{3}$ & Yacimiento B & 28.42 & 0.79 \\
$\mathbf{4}$ & Yacimiento C & 2.55 & 0.02 \\
\hline
\end{tabular}

\section{RANIKING DE ESTABILIDAD}

Para evaluar la estabilidad se construyeron modelos 2D con celdas de 1 metro de espesor y se consideraron los mismos parámetros de control que en el modelo 1D sin re-presurización. El ranking se construyó con los volúmenes porosos inyectados hasta el tiempo de irrupción (Tabla 10). Nuevamente, el Yacimiento $A$ presenta los mejores resultados, esta vez como resultado de su espesor, ya que al tener un espesor de formación más delgado que los demás yacimientos, además de unas buenas condiciones de transmisibilidad reduce significativamente los efectos producidos por el fenómeno de segregación gravitacional

\section{RANKING DE FACTOR DE RECOBRO}

Como lo sugiere Niz-Velásquez et al. (2014) en su metodología se construyó un ranking absoluto con el factor de recobro a un tiempo de corte estipulado (10 años). Para la construcción de dicho ranking se tomaron los resultados del modelo 2D, encontrando que los yacimientos $\mathrm{A}$ y $\mathrm{D}$ son quienes tienen la mejor eficiencia de recuperación mediante el desplazamiento inmiscible de gas.

Con base en lo observado tras la construcción de cada uno de los rankings para la priorización de yacimientos candidatos al proceso LOAI, se identifica claramente que para el caso de estudio presentado el Yacimiento $A$ es el que reúne las mejores condiciones para la implementación del proceso. Sin embargo, no se puede 
olvidar que la metodología implementada no tiene en cuenta efectos térmicos, composicionales o cinéticos; por lo tanto, posterior a la selección del yacimiento candidato y antes de implementar el proceso a escala de campo es necesario estudiar estos fenómenos a escala experimental y numérica.

Tabla 10. Ranking de estabilidad del desplazamiento inmiscible.

\begin{tabular}{cccc}
\hline Prioridad & Yacimiento & $\begin{array}{c}\text { Volúmenes Porosos } \\
\text { Inyectados }\end{array}$ & $\begin{array}{c}\text { Tiempo de } \\
\text { irrupción, días }\end{array}$ \\
\hline $\mathbf{1}$ & Yacimiento A & 0.068 & 90 \\
$\mathbf{2}$ & Yacimiento D & 0.047 & 92 \\
$\mathbf{3}$ & Yacimiento C & 0.020 & 1647 \\
$\mathbf{4}$ & Yacimiento B & 0.018 & 31 \\
\hline
\end{tabular}

Tabla 11. Ranking de Factor de Recobro.

\begin{tabular}{ccc}
\hline Prioridad & Yacimiento & Factor de Recobro @ $\mathbf{1 0}$ años , \% \\
$\mathbf{1}$ & Yacimiento D & 35 \\
$\mathbf{2}$ & Yacimiento A & 22 \\
$\mathbf{3}$ & Yacimiento B & 11 \\
$\mathbf{4}$ & Yacimiento C & 6 \\
\hline
\end{tabular}

\section{CONCLUSIONES}

La metodología propuesta por Niz-Velásquez et al. (2014) se constituye en una herramienta sencilla para la priorización de yacimientos de crudo liviano con buenas características para la inyección de aire, sin la ejecución de estudios experimentales y/o la realización de estudios de simulación numérica robustos. No obstante, previo a la implementación de la técnica en campo se debe estudiar el proceso a escalas experimental y numérica.

\section{AGRADECIMIENTOS}

Los autores agradecen a Ecopetrol S.A. por permitir la publicación de este documento.

\section{REFERENCIAS}

1. Greaves, M., Ren S. R. y Rathbone, R. (1998). Air Injection Technique (LTO Process) for IOR from Light Oil Reservoirs: Oxidation rate and displacement studies. SPE 40062 presentado en SPE/DOE Improved Oil Recovery Symposium, Tulsa, Estados Unidos.

2. Hughes, B. L. y Sarma, H. K. (2006). Burning Reserves for Greater Recovery? Air Injection Potential In Australian Light Oil Reservoirs. SPE 101099 presentado en SPE Asia Pacific Oil \& Gas Conference and Exhibition, Adelaide, Australia.
3. Montes, A. R., Gutierrez, D., Moore, R. G., Mehta, S. A. y Ursenbach, M. G. (2010). Is HighPressure Air Injection (HPAI) Simply a FlueGas Flood?. Journal of Canadian Petroleum Technology, Vol. 49 (2) p. $57-63$.

4. Niz-Velásquez, E., Trujillo, M. L., Delgadillo, C. y Padilla, J. (2014). A Methodology for Screening and Ranking of Reservoirs for Light Oil Air Injection Implementation. SPE 169951 presentado en SPE Biennial Energy Resources Conference, Port of Spain, Trinidad \& Tobago.

5. Turta, A. T. y Singhal, A. K. (2001). Reservoir Engineering Aspects of Light-Oil Recovery by Air Injection. SPE Reservoir Evaluation \& Engineering.

\section{ANEXOS}

Tabla 11. Características del Yacimiento A

\begin{tabular}{|cc|}
\hline Propiedad & Valor \\
\hline Tope de la formación [ft] & 6900 \\
\hline Espesor [ft] & 15 \\
\hline Temperatura [ $\left.{ }^{\circ} \mathrm{F}\right]$ & 207 \\
\hline Presión inicial [psi] & 1580 \\
\hline Presión de burbuja [psi] & 47 \\
\hline Permeabilidad i,j [md] & 2500 \\
\hline Permeabilidad k [md] & 250 \\
\hline Porosidad [\%] & 25 \\
\hline Saturación de aceite [\%] & 75 \\
\hline Saturación de agua [\%] & 25 \\
\hline Gravedad del aceite [ $\left.{ }^{\circ} \mathrm{API}\right]$ & 29.6 \\
\hline Buzamiento $\left[{ }^{\circ}\right.$ ] & 0 \\
\hline
\end{tabular}

Tabla 12. Características del Yacimiento B

\begin{tabular}{|cc|}
\hline Propiedad & Valor \\
\hline Tope de la formación [ft] & 9700 \\
\hline Espesor [ft] & 36 \\
\hline Temperatura [ $\left.{ }^{\circ} \mathrm{F}\right]$ & 230 \\
\hline Presión inicial [psi] & 2400 \\
\hline Presión de burbuja [psi] & 2425 \\
\hline Permeabilidad i,j [md] & 120 \\
\hline Permeabilidad $\mathrm{k}[\mathrm{md}]$ & 12 \\
\hline Porosidad [\%] & 13 \\
\hline Saturación de aceite [\%] & 70 \\
\hline Saturación de agua [\%] & 9 \\
\hline Gravedad del aceite $\left[{ }^{\circ} \mathrm{API}\right]$ & 30.7 \\
\hline Buzamiento $\left[{ }^{\circ}\right.$ ] & 12.5 \\
\hline
\end{tabular}


Tabla 13. Características del Yacimiento C

\begin{tabular}{|cc|}
\hline Propiedad & Valor \\
\hline Tope de la formación [ft] & 6800 \\
\hline Espesor [ft] & 105 \\
\hline Temperatura [ $\left.{ }^{\circ} \mathrm{F}\right]$ & 143 \\
\hline Presión inicial [psi] & 1469 \\
\hline Presión de burbuja [psi] & 790 \\
\hline Permeabilidad i,j [md] & 78 \\
\hline Permeabilidad k [md] & 7.8 \\
\hline Porosidad [\%] & 18.5 \\
\hline Saturación de aceite [\%] & 54.5 \\
\hline Saturación de agua [\%] & 45.5 \\
\hline Gravedad del aceite [ $\left.{ }^{\circ} \mathrm{API}\right]$ & 71 \\
\hline Buzamiento $\left[{ }^{\circ}\right.$ ] & 37.5 \\
\hline
\end{tabular}

Tabla 14. Características del Yacimiento D

\begin{tabular}{cc}
\hline Propiedad & Valor \\
\hline Tope de la formación [ft] & 8300 \\
\hline Espesor [ft] & 35 \\
\hline Temperatura [ $\left.{ }^{\circ} \mathrm{F}\right]$ & 204 \\
\hline Presión inicial [psi] & 2100 \\
\hline Presión de burbuja [psi] & 695 \\
\hline Permeabilidad i,j [md] & 559 \\
\hline Permeabilidad $\mathrm{k}[\mathrm{md}]$ & 55.9 \\
\hline Porosidad [\%] & 13.5 \\
\hline Saturación de aceite [\%] & 71 \\
\hline Saturación de agua [\%] & 29 \\
Gravedad del aceite [ $\left.{ }^{\circ} \mathrm{API}\right]$ & 26.5 \\
\hline Buzamiento $\left[{ }^{\circ}\right.$ ] & 5 \\
\hline
\end{tabular}

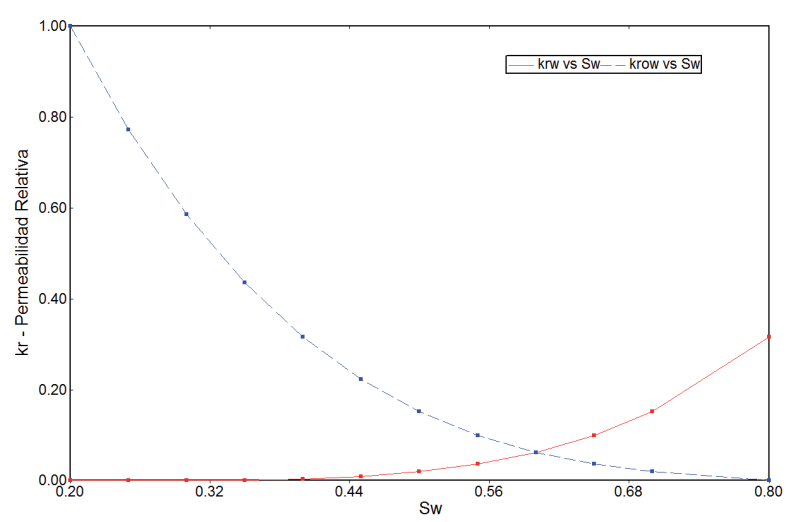

Figura 3. Curvas permeabilidad relativa sistema agua aceite, Yacimiento A.

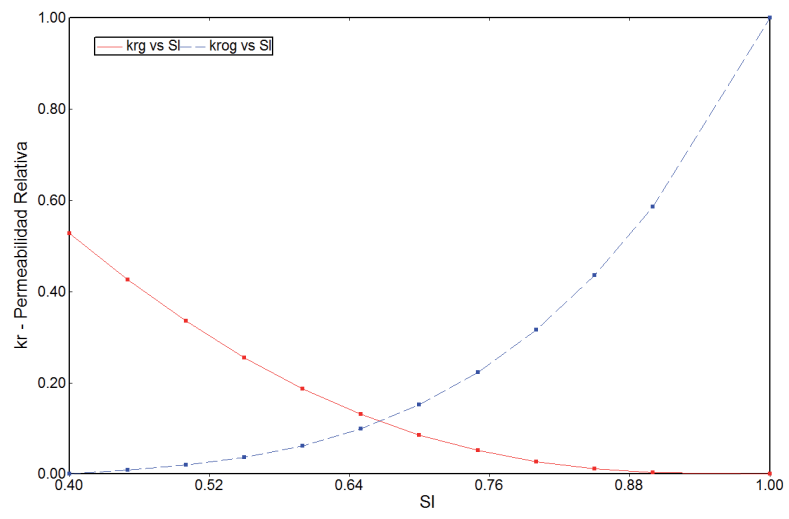

Figura 4. Curvas permeabilidad relativa sistema liquido gas, Yacimiento A.

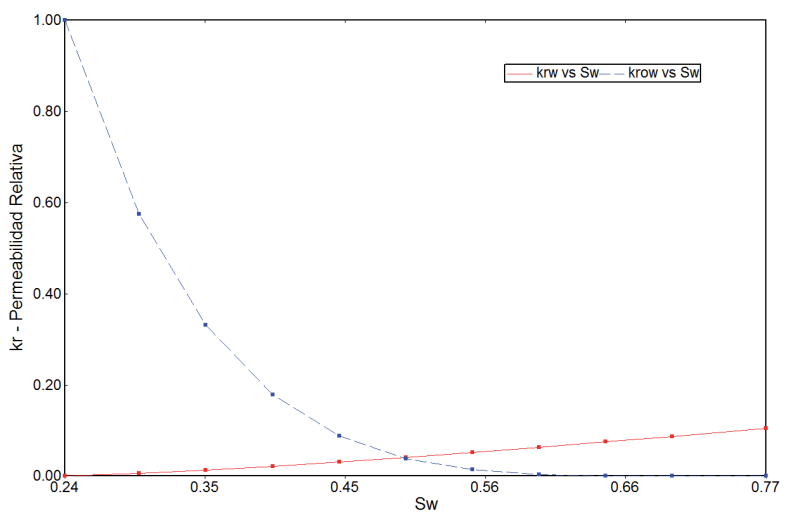

Figura 5. Curvas permeabilidad relativa sistema agua aceite, Yacimiento B.

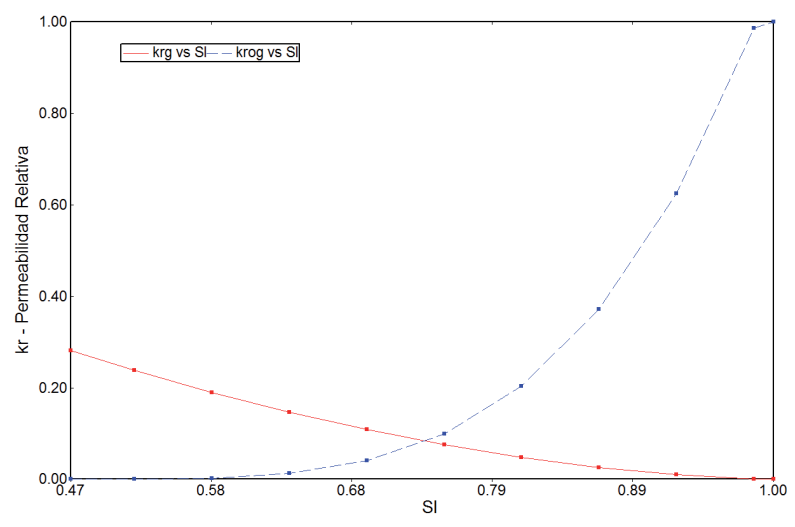

Figura 6. Curvas permeabilidad relativa sistema liquido gas, Yacimiento B. 


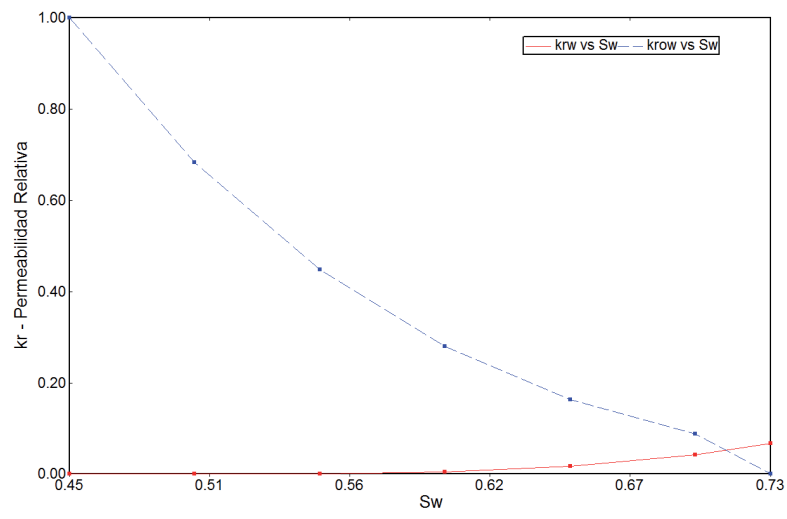

Figura 7. Curvas permeabilidad relativa sistema agua aceite, Yacimiento $\mathrm{C}$.

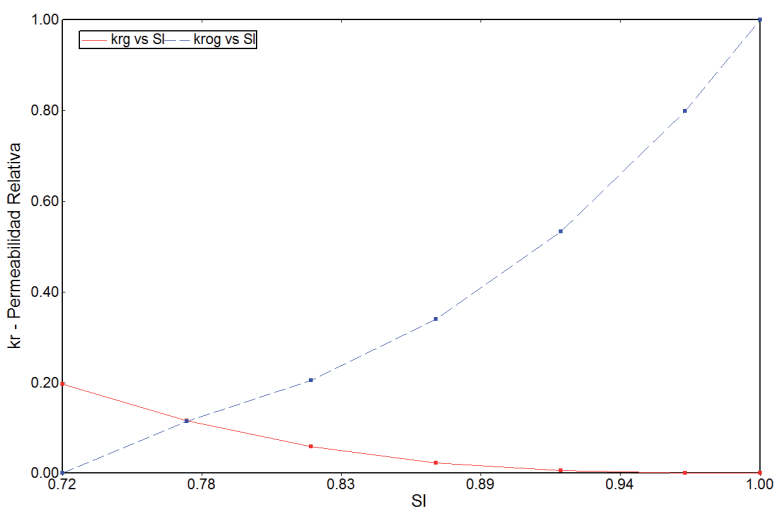

Figura 8. Curvas permeabilidad relativa sistema liquido gas, Yacimiento $\mathrm{C}$.

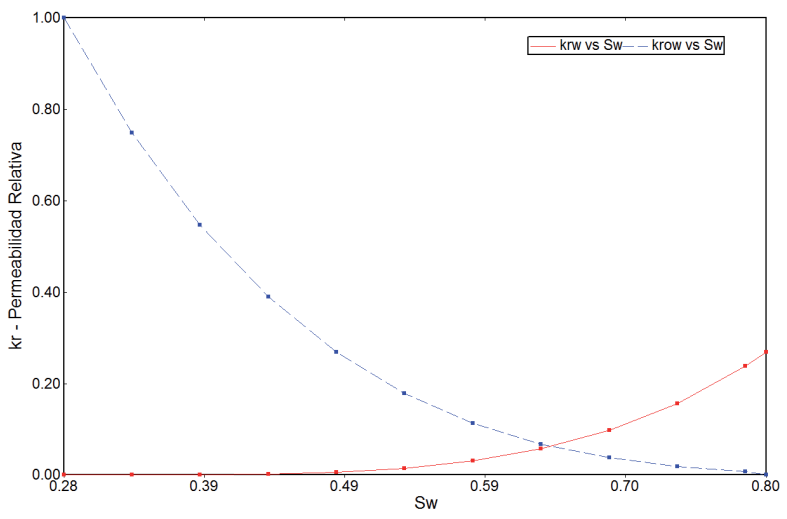

Figura 9. Curvas permeabilidad relativa sistema agua aceite, Yacimiento D.

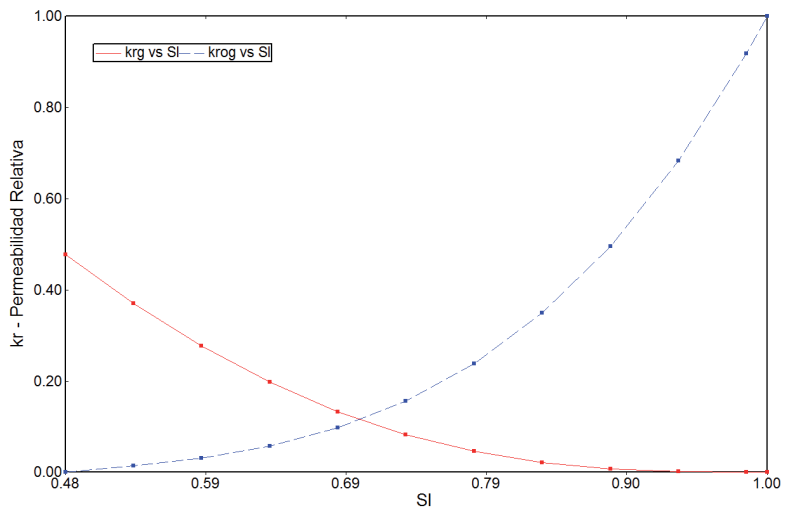

Figura 10. Curvas permeabilidad relativa sistema liquido gas, Yacimiento D.

Recepción: 5 de febrero de 2017

Aceptación: 25 de abril de 2017 